\title{
De la grammaire pure logique à la grammaire philosophique : Husserl et Wittgenstein
}

\author{
Par CHARLOTTE GAUVRY \\ Université de Liège
}

Dans la quatrième Recherche logique, dans l'héritage des travaux de Bernard Bolzano, Edmund Husserl défend la thèse d'après laquelle le sens est structuré selon les lois syntaxiques de la "grammaire pure logique», indépendamment de toute considération d'ordre ontologique ou sémantique. Ces «lois de signification» sont en effet des lois de compatibilité et non de correction ou de contradiction. Ce sont des «lois a priori, faisant abstraction de la validité objective (de la vérité ou encore de l'objectivité réelles [reale] ou formelles) ${ }^{1}$. Aussi, loin de reconduire le sens à ses conditions de vérité, dans la quatrième Recherche logique, Husserl énonce la thèse de l'autonomie des lois syntaxiques et distingue fermement le régime du «sens » [Sinn] du régime des objets (ou des références [Bedeutungen]) et de celui de la «vérité ». C'est l'hypothèse d'une autonomie des lois syntaxiques que nous souhaitons ici interroger.

À cette fin, une comparaison avec le traitement que Ludwig Wittgenstein réserve à la syntaxe formelle puis à la grammaire philosophique nous paraît opératoire. Il existe une certaine parenté entre la grammaire pure logique husserlienne et la syntaxe formelle wittgensteinienne déployée dans le Tractatus logico-philosophicus. Mais Wittgenstein, du moins celui que l'on appelle le "second Wittgenstein", apporte plusieurs arguments pour contester le caractère a priori et formel des lois grammaticales. Qui plus est, Wittgenstein accomplit un pas décisif par rapport à Husserl à partir du début des années 1930. Comme Husserl en un sens, il accorde certes la priorité aux règles grammaticales. Mais son analyse tend à montrer que les règles séman-

${ }^{1}$ E. Husserl, Recherche logique IV, dans Recherches logiques, t. II/2 : Recherches pour la phénoménologie et la théorie de la connaissance, Paris, PUF, 2002, p. 85. 
tiques elles-mêmes sont gouvernées par des règles grammaticales. Aussi, loin d'insister sur l'hétérogénéité des règles grammaticales et sémantiques, ses recherches contribuent à réviser la distinction traditionnelle entre la syntaxe et la sémantique. En montrant que l'on ne peut fixer la référence d'un concept ou évaluer la valeur de vérité d'une proposition qu'en considérant au préalable les règles grammaticales qui règlent son usage, Wittgenstein souligne la sensibilité à l'usage, et donc à la grammaire, de la sémantique des concepts. Il défend en conséquence la thèse d'après laquelle les règles grammaticales, qui sont déterminés en fonction des contextes d'application des concepts, règlent l'application de tous les concepts, tant et si bien que c'est le propre des concepts, de ce que l'on pourra appeler le « conceptuel », que d'être grammaticalement déterminés. C'est la portée de cette thèse que nous interrogerons pour finir.

\section{Husserl et Wittgenstein}

\subsection{De la grammaire pure logique à la syntaxe formelle}

L'un des principaux résultats de la quatrième Recherche logique de Husserl consiste à distinguer le plan du sens du plan de la référence objective et de celui de la vérité. La tâche de la " grammaire pure logique », ou de l'étude de la « morphologie des catégories », consiste en effet à déceler les «non-sens » (Unsinne) qui contreviennent à la possibilité même de la "signification unitaire», pour reprendre les termes de Husserl ${ }^{1}$. Comme l'illustrent ses célèbres exemples, des énoncés comme « un rond ou », « un homme et est » ou «vert est ou » ${ }^{2}$ sont des non-sens, non pas en tant qu'ils n'ont pas de référence, qu'ils sont faux ou qu'ils violent les règles de contradiction, mais en tant qu'ils ne respectent pas les règles minimalement requises pour l'expression d'un sens unitaire. Ils n'ont pas même d'objet. On pourrait discuter de la pertinence de la méthode husserlienne d'identification des différentes « catégories de signification » et douter du caractère a priori des règles grammaticales censées les combiner et trancher définitivement ce qui

${ }^{1}$ En fidélité à la langue allemande, Husserl use ici indifféremment des concepts de «sens » (Sinn) et de «signification/référence » (Bedeutung). Ce problème terminologique mis à part, il est clair que la tâche de la grammaire pure logique est d'étudier la morphologie du «sens », sans considération aucune pour ce qui «porte [...] atteinte à l'objectivité ou à la vérité des significations entières ", Recherche logique IV, ibid., p. 121.

${ }^{2}$ Ibid. 
est de l'ordre du sens et du non-sens. Il reste cependant indéniable que l'analyse husserlienne présente le mérite d'énoncer clairement la thèse de la priorité logique des lois de signification exclusivement grammaticales.

De ce strict point de vue, il est possible de proposer un premier rapprochement prudent avec l'analyse de la syntaxe logique du Tractatus logico-philosophicus de Wittgenstein ${ }^{1}$. Le Tractatus logico-philosophicus, comme son nom l'indique, accorde le primat à l'analyse logique de la langue en général, et en particulier à l'étude de la syntaxe des propositions. Le traité soutient qu'il convient de purifier la langue ordinaire des confusions qui s'y immiscent au moyen d'une langue symbolique qui obéit aux règles clarifiées de la syntaxe logique ${ }^{2}$. Or Wittgenstein considère ces règles syntaxiques comme purement formelles et comme ne donnant aucune indication de contenu : "Les propositions logiques décrivent l'échafaudage du monde, ou plutôt elles le figurent. Elles ne "traitent" de rien» $(6.124)^{3}$. Qui plus est, si l'on se réfère à la proposition 3.3 du Tractatus, il apparaît que la tâche de la syntaxe logique est d'organiser le régime du « sens » des symboles et non pas celui de leur «référence» [Bedeutung]. Comme l'énonce en effet Wittgenstein, «dans la syntaxe logique, la Bedeutung d'un signe n'a jamais le droit de jouer aucun rôle $»^{4}$. Aussi, les lois de la syntaxe logique, tout comme les lois de la "grammaire pure logique» husserlienne, font abstraction du «type d'objet» qui est rattaché au discours. Ce ne sont pas des lois ontologiques; ce sont exclusivement les règles logiques de l'organisation du sens et non pas de la référence ${ }^{5}$.

Aussi, Husserl comme Wittgenstein, dans les deux œuvres citées du moins, soutiennent de concert que les règles grammaticales ou syntaxiques sont des règles autonomes qui ont la priorité sur les règles ontologiques. On

1 Pour une lecture similaire, à laquelle l'auteur ne souscrit cependant plus, cf. J. Benoist, « Grammaires pures logiques », dans J. Benoist et S. Laugier (éds.), Husserl et Wittgenstein. De la description de l'expérience à la phénoménologie linguistique, Hildesheim, Olms, 2004.

2 L. Wittgenstein, Tractatus logico-philosophicus, Paris, Gallimard, 1993, 3.325, p. 47. (Tractatus par la suite)

${ }^{3}$ Ibid., p. 101.

${ }^{4}$ Ibid., p. 47.

${ }^{5} \mathrm{Il}$ y a donc selon nous des règles syntaxiques qui déterminent le sens dans le Tractatus, contrairement à ce que défendent certains lecteurs dits "austères " du Tractatus. Cf. p.ex. C. Diamond, "Ce que le non-sens pourrait être », dans Id., $L$ 'esprit réaliste, trad. fr. par E. Halais et J.-Y. Mondon, Paris, Presses Universitaires de France, 2004 et J. Conant, « Wittgenstein. On Meaning and Use », Philosophical Investigations 21, 1998. 
ne peut cependant pas pousser beaucoup plus loin le rapprochement. Là où Husserl distingue très clairement les lois de compatibilité grammaticale qui règlent la morphologie des catégories des lois de contradiction, Wittgenstein semble suggérer pour commencer, du moins à l'époque du Tractatus, qu'une proposition syntaxiquement bien formée est une proposition non contradictoire. C'est en effet en tant qu'elles sont contradictoires qu'il faut exclure du règne du sens logique les propositions qui violent les lois d'exclusion des couleurs ${ }^{1}$. Plus généralement, pour expliquer ce premier différend entre les deux positions, il convient d'accorder toute attention au traitement que la grammaire pure husserlienne et la syntaxe logique wittgensteinienne réservent à la question de la grammaire des couleurs.

\subsection{La grammaire des couleurs}

La question de la "grammaire des couleurs » est une question hautement débattue $^{2}$, au moins depuis les travaux de Johann Wolfgang von Goethe. Ce champ de recherche consiste à vérifier s'il existe des lois grammaticales (parfois appelées "relations internes ») qui régulent l'ordonnancement des couleurs : par exemple le fait que le violet se situe non pas entre le jaune et le rouge mais entre le bleu et le rouge ou qu'il est possible de parler d'un orange qui tire sur le rouge mais pas d'un orange qui tire sur le violet, etc. L'un des enjeux de l'analyse est de préciser la nature de ces lois (empiriques ou a priori, formelles ou matérielles, conceptuelles ou phénoménales, etc.). Or ces questions se sont essentiellement focalisées sur deux problèmes : le problème de l'exclusion des couleurs (Peut-on trouver deux couleurs différentes en même temps et au même endroit ?) et de l'incompatibilité des couleurs (Cela a-t-il un sens de parler de vert-bleuâtre ou de rouge-jaunâtre par exemple ?). Il s'agit de questions qui traversent tout le corpus de Wittgenstein, de la proposition 6.3751 du Tractatus que nous venons de citer aux derniers écrits sur les Remarques sur les couleurs de 1950-51.

On sait que Husserl s'est intéressé pour sa part aux énoncés mobilisant des exclusions de couleur comme « un objet n'est pas rouge et vert en même temps ». Rappelons que Husserl, en reprenant la définition de l'analyticité

${ }^{1}$ Cf. L. Wittgenstein, Tractatus, op. cit., 6.3751, p. 109 : « C'est impossible, et même logiquement impossible, car c'est la structure logique de la couleur qui l'exclut ».

${ }^{2}$ Sur cette question, nous renvoyons à l'excellent article de Denis Seron du présent numéro. 
proposée dans le paragraphe 148 de la Wissenschaftslehre de Bolzano, considère qu'un énoncé analytique est un énoncé dont la valeur de vérité des propositions est complètement indépendante de la nature concrète de ses composants :

Nous pouvons définir des propositions analytiquement nécessaires comme étant celles qui comportent une vérité pleinement indépendante de la nature concrète particulière de leurs objectivités [...] ainsi que de la facticité éventuelle du cas donné et de la valeur de la position éventuelle d'existence ${ }^{1}$.

Un énoncé analytique est donc un énoncé dont on peut remplacer, par lois de substitution, tous les concepts par d'autres salva veritate, leur forme logique restant invariable. Selon cette définition, un énoncé comme « un objet n'est pas rouge et vert en même temps » n'est précisément pas un énoncé analytique pour Husserl. Car si l'on substitue au concept de « rouge» celui de "grand » par exemple, l'énoncé peut devenir faux. Il s'agit donc d'un énoncé qui ne répond précisément pas aux seules règles de la grammaire pure logique mais à des « lois synthétiques $a$ priori »: des lois qui impliquent des « concepts concrets» qui «se fondent sur la nature spécifique des contenus $»^{2}$.

Pour Wittgenstein en revanche, du moins jusqu'en 1929, un énoncé de ce type est régulé par des lois a priori qui sont nécessairement (exclusivement) des lois analytiques. Wittgenstein reproche en conséquence aux phénoménologues (à Husserl au premier chef) d'avoir postulé l'existence de ces « lois synthétiques a priori». Dans l'entretien de Wittgenstein avec Schlick de 1929, Wittgenstein explique en effet que dans un énoncé comme « rouge et vert ne peuvent pas être au même point au même moment ", «le mot "peut" est manifestement un concept grammatical (logique) et non pas un concept chosique $»^{3}$. Selon Wittgenstein, un énoncé de ce type ne procède pas par une généralisation empirique. Quand bien même il ne violerait pas les lois de contradiction, il ne pourrait exprimer qu'une impossibilité «grammaticale », le terme étant entendu ici comme un synonyme de « logique». Pour reprendre ses exemples, avec un énoncé empirique comme « je n'ai pas mal au ventre », il est empiriquement possible d'envisager que j'aie un jour « mal

${ }^{1}$ E. Husserl, Recherche logique III, dans Recherches logiques, t. II/2, op. cit., § 12, p. 39.

${ }^{2}$ Ibid., p. 39-40.

${ }^{3}$ G. Baker, B. McGuinness et A. Soulez (éds.), Dictées de Wittgenstein à Waismann et pour Schlick, Paris, Presses Universitaires de France, 1997, vol. 1, «AntiHusserl ». 
au ventre $»$ : il est donc possible d'envisager la vérité du négatif de l'énoncé. Or ce n'est précisément pas le cas d'un énoncé logique comme « un objet n'est pas rouge et vert en même temps ». Car il est logiquement impossible, selon Wittgenstein, qu'un objet soit rouge et vert en même temps. C'est encore la position qui est défendue dans les Remarques sur la forme logique de 1929 à propos des lois de compatibilité, présentées comme des lois qui « sont en quelque sorte des tautologies » :

Une nuance de couleur ne peut pas avoir simultanément deux degrés différents de luminosité ou de rougeur, un son ne peut avoir deux intensités différentes, etc. Le point important est ici que ces remarques n'expriment pas une expérience, mais sont en quelque sorte des tautologies ${ }^{1}$.

Ce serait donc un non-sens de dire que « deux couleurs sont ensemble en un même lieu du champ visuel » ou qu'une couleur a deux degrés de luminosité différents, dans la mesure où ces deux propositions sont logiquement inconsistantes.

Moritz Schlick, dans son texte de 1932, Forme et contenu ${ }^{2}$, durcit l'opposition en affirmant que les énoncés analytiques régulés par la grammaire logique ne mobilisent nécessairement que des concepts purement formels et qu'à ce titre, des énoncés comme « toute couleur a une extension » ou « une surface ne peut être rouge et verte au même moment et à la même place » ne sont pas des énoncés synthétiques mais bien des énoncés logiques (ou grammaticaux) en ceci qu'ils n'énoncent rien sur le "contenu» de l'énoncé et qu'ils s'imposent comme des évidences logiques (des tautologies) :

La proposition n'est rien d'autre qu'une tautologie qui révèle la manière (ou la forme) dans laquelle les termes « rouge » et « vert » sont utilisés. L'incompatibilité entre les deux est due non pas à quelque antagonisme mystérieux entre deux essences réelles, deux espèces de contenu, mais à la structure interne des deux concepts " vert » et « rouge ». Une surface ne peut être rouge et verte pour exactement la même raison qui rend impossible pour un homme grand d'être en même temps un homme petit. [...] Nous savons cela a priori, c'est-à-dire sans consulter l'expérience, et nous ne le considérons pas comme étant une assertion qui transmet une connaissance concernant l'essence d' «homme » ou même l'essence de « longueur ». Nous savons que sa vérité

${ }^{1}$ L. Wittgenstein, Remarques sur la forme logique, Mauvezin, TER, 1985, p. 26.

${ }^{2} \mathrm{M}$. Schlick, Forme et contenu. Une introduction à la pensée philosophique, Marseille, Agone, 2003. 
est purement formelle. [...] C'est comme dans tous les cas de structure interne, une question de règles grammaticales ${ }^{1}$.

En conséquence, pour Schlick, dont l'exposé se veut fidèle à l'enseignement du Tractatus, les seules lois a priori sont nécessairement analytiques (et formelles). C'est ce qu'il nomme « règles grammaticales »: les règles de « la structure interne de deux concepts "vert" et "rouge" ». La définition de la « règle grammaticale » de la syntaxe logique se précise en conséquence. Si l'on en croit Schlick, il s'agit de règles purement formelles qui font abstraction de toute considération de contenu, qui s'imposent avec évidence et qui relèvent de la structure interne des concepts formels. Ce sont donc tout sauf des lois eidétiques matérielles. Le désaccord avec Husserl est précisé sur ce point.

Une remarque cependant. Ce désaccord concerne moins la conception de la "grammaire» de Husserl et de Wittgenstein que l'extension qu'ils accordent au concept. Pour reprendre une formule de Bouveresse qui commente le texte de Schlick, les règles grammaticales «ne formulent aucune assertion à propos de la réalité » et «ne communiquent par conséquence aucune connaissance ou information dignes de ce nom ; elles peuvent être dites, en ce sens-là, "tautologiques" et ne sont même pas vraiment des propositions $»^{2}$. Or, pour le Husserl de la quatrième Recherche logique aussi, les règles grammaticales sont exclusivement a priori et formelles en ce qu'elles font abstraction de toute considération de « contenu ». Le désaccord entre les deux auteurs porte alors sur l'extension du formel ou, plutôt de l'analyticité. Comme nous l'avons rappelé, pour Husserl, seule une proposition qui satisfait le test leibnizien de «substituabilité " peut être qualifiée de proposition analytique. Aussi, pour être charitable avec Husserl et réévaluer les critiques de Schlick, on pourrait aller jusqu'à dire que du point de vue de Husserl, Wittgenstein propose une extension injustifiée du rôle de la grammaire pure, en qualifiant de " grammaticale » ou « logique » toute loi $a$ priori. C'est ce que refuse précisément de faire Husserl en stipulant pour sa part que la logique de certains énoncés, ceux qui mobilisent ce qu'il appelle des « concepts concrets », relèvent plutôt d'une « logique du contenu » dont les lois d'organisation sont eidétiques. Selon lui, il faudrait donc clairement distinguer les lois grammaticales, les lois de contradiction (ce que concède en un sens Wittgenstein) et les lois eidétiques.

${ }^{1}$ Ibid., p. 148-149.

${ }^{2}$ J. Bouveresse, Les lumières des positivistes. Essai VI, Marseille, Agone, 2011, p. 218. 
Or c'est loin d'être la voie empruntée par Wittgenstein. Avec la reformulation du concept de «grammaire » qui s'opère à partir de 1930, le philosophe propose au contraire une extension supplémentaire de la portée des règles grammaticales. En renonçant au modèle de la syntaxe logique, et en désolidarisant le concept de " grammaire » de celui de «syntaxe », Wittgenstein propose en effet une extension de la portée de la grammaire à tout le champ du conceptuel et insiste qui plus est sur la sensibilité à l'usage des règles de compatibilité, selon un mouvement qui s'initie dès les Remarques sur la forme logique.

\section{La grammaire philosophique chez Wittgenstein}

Il est bien connu que le retour à la philosophie de Wittgenstein à Cambridge se manifeste par un revirement assez fondamental de sa pensée. S'il ne faut pas pour autant sous-estimer la profonde continuité de sa pensée, il est indéniable que le concept même de "grammaire » subit une profonde inflexion. Le programme qui était encore celui de la «syntaxe logique universelle » dans le Tractatus est abandonné à plusieurs titres.

Dans les textes de 1932-34, légèrement ultérieurs aux Remarques sur la forme logique de 1929, rassemblés sous le titre La Grammaire philosophique, s'initie l'idée que les règles grammaticales ne sont précisément plus des règles strictement formelles. C'est par exemple déjà manifeste au paragraphe $\S 27$ de la Grammaire qui analyse un exemple proche de celui dont discutait déjà Wittgenstein avec Schlick en 1929 :

27. Le nom que je donne à un corps, - à une forme, - à une couleur, - à une longueur, change toutes les fois de grammaire. («A » dans "A est jaune » a une grammaire différente suivant que A est un corps ou qu'il est la surface d'un corps ; par exemple cela a un sens de dire que le corps est jaune de part en part, mais cela n'en a pas de le dire de sa surface.) ${ }^{1}$

Le paragraphe confirme l'idée qu'un énoncé qui mobilise des concepts de couleur comme « A est jaune » est déterminé par des règles grammaticales et que leur application doit obéir à des règles. C'est bien la première idée fondamentale que sous-tend le concept de "grammaire»: la grammaire procède selon des règles. Cependant, ce paragraphe 27 introduit une rupture importante par rapport à l'analyse précédente dans la mesure où il suggère qu'une telle règle ne peut pas faire abstraction du «contenu » de l'énoncé.

${ }^{1}$ L. Wittgenstein, Grammaire philosophique, Paris, Gallimard, 1980, p. 90. 
«A est jaune », nous dit en effet Wittgenstein, « a une grammaire différente suivant que A est un corps ou qu'il est la surface d'un corps ». Wittgenstein soutient désormais que les règles d'application des concepts, en l'occurrence de « $\mathrm{A}$ », doivent prendre en compte le « contenu » des concepts, qui ne sont donc plus seulement considérés comme des concepts formels. Les règles d'application des concepts ne relèvent donc plus de leur « structure interne ». Pour le dire autrement, Wittgenstein soutient dès lors la thèse que les règles grammaticales qui départagent le sens et le non-sens ne sont plus déterminées par les règles de la syntaxe logique ${ }^{1}$.

L'analyse wittgensteinienne qui s'initie à partir de 1932 insiste également sur le fait que ces lois ne sont pas non plus des lois transcendantales qui anticipent l'énonciation. C'est ce que confirme déjà la Grammaire philosophique, par exemple au $\S 44$ : «la grammaire est le livre de comptes du langage : ce qu'on doit y trouver ce ne sont pas les impressions qui accompagnent le langage, mais les transactions linguistiques réelles $»^{2}$. La grammaire enregistre donc les règles des échanges linguistiques effectifs et ne les prescrit pas en amont. Les Recherches philosophiques, par exemple au célèbre $\S 496$ sont plus claires encore : «La grammaire ne dit pas comment le langage doit être construit pour atteindre son but, pour agir de telle ou telle manière sur les hommes. Elle décrit seulement l'emploi des signes, et ne l'explique d'aucune façon $»^{3}$. La description en question est malgré tout orientée et réglée par les problèmes à dissoudre.

Pour mesurer la rupture opérée, il est encore une fois utile d'examiner le traitement que Wittgenstein réserve aux concepts de couleur, par exemple dans son texte bien plus tardif de 1948 : les Remarques sur la philosophie de la psychologie. Par sa méthode de variation habituelle, en imaginant de nouveaux jeux de langage, fictifs mais plausibles, Wittgenstein suggère clairement que les règles grammaticales qui gouvernent l'application des concepts de couleur $1 /$ ne relèvent pas de lois de contradiction logique et $2 /$ sont sensibles au contenu et surtout au contexte d'usage des concepts :

\footnotetext{
${ }^{1}$ Notons cependant que ce point est controversé. C'est du moins l'objet de la controverse célèbre qui opposa Gordon Baker à Peter Hacker, après des années de collaboration. Là où Hacker soutient que, chez le second Wittgenstein, la grammaire est réglée par les critères de la syntaxe logique qui régulent son usage correct, Baker s'est dissocié de l'analyse à laquelle il avait initialement souscrit pour défendre que ces critères (ceux qui démarquent le sens du non-sens) sont sensibles à l'usage et contextuellement déterminés. Nous souscrivons à cette deuxième interprétation.

${ }^{2}$ Ibid., p. 119.

${ }^{3}$ L. Wittgenstein, Recherches philosophiques, Paris, Gallimard, 2004, p. 198.
} 
$\S$ 658. Imagine différents cas. (1) Certaines couleurs liées à certaines formes. Le rouge circulaire, le vert quadrangulaire, etc. (2) Les couleurs ne peuvent être produites. On ne peut colorer les choses. (3) Telle couleur toujours liée à une odeur désagréable ou à un poison. (4) Le daltonisme beaucoup plus fréquent que chez nous. (5) Différentes nuances de gris sont chose fréquente ; toutes les autres couleurs extrêmement rares (6) Nous pouvons reproduire de mémoire un grand nombre de nuances de couleur. Si notre système de numération est lié au nombre de nos doigts, pourquoi notre système de couleur ne serait-il pas lié à la façon particulière dont les couleurs apparaissent ? (7) Une couleur apparaît toujours dans un passage graduel vers une autre couleur. (8) Les couleurs apparaissent toujours dans l'ordre de l'arc-en-ciel ${ }^{1}$.

Dans tous ces exemples, il ne s'agit pas de nier le fait qu'il existe une grammaire des concepts de couleurs et donc des lois d'association, d'exclusion ou d'incompatibilité. Il s'agit plutôt de suggérer la sensibilité à l'usage et à l'occasion de ces règles. Pour prendre un cas concret - on pourrait en chercher de nombreux autres -, tout habitué des pistes de ski classera spontanément la couleur « rouge » entre le «bleu » et le «noir» au moment de s'engager sur une piste rouge. C'est dire que les règles d'organisation des concepts de couleur ne sont pas déterminées a priori, en faisant abstraction des considérations contextuelles: l'organisation des concepts de couleur n'est pas seulement tributaire de l'ordre chromatique mais bien de règles d'usage contextuellement fixées. Or la leçon wittgensteinienne consiste précisément à montrer que les lois d'organisation des concepts de couleur, et plus généralement de tous les concepts, sont sensibles à l'usage qu'on en fait en contexte.

\section{La grammaire des concepts}

Pour conforter cette lecture de la grammaire chez le second Wittgenstein, il est précieux de s'appuyer sur la lecture contextualiste qu'en propose Charles Travis qui présente le mérite d'insister sur la « sensibilité à l'occasion» de la grammaire, et sur son objet spécifique, le «conceptuel». Charles Travis reprend à son compte bon nombre des analyses du second Wittgenstein. Dans son texte " Jusqu'où va la raison » par exemple, il propose une analyse très proche de celle du $§ 658$ des Remarques sur la philosophie de la psychologie précédemment cité et en radicalise le propos. À propos de la question des

${ }^{1}$ L. Wittgenstein, Remarques sur la philosophie de la psychologie II, Mauvezin, TER, 1994, p. 131-132. 
règles «d'incompatibilité des couleurs », Travis soutient qu'il ne peut s'agir de règles de contradiction logique et va jusqu'à imaginer un contexte où « une chose peut être à la fois bleue et biolette»:

«Est-ce que la couleur s'analyse absolument en une seule gamme de couleurs : les couleurs? » En un sens, non. On peut diviser le spectre des couleurs de diverses façons. Mais aucune façon n'en exclut une autre. Imaginons que le biolet soit une couleur qui corresponde uniquement à certaines teintes de violet et à certaines teintes de bleu. Alors une chose peut être à la fois bleue et biolette ${ }^{1}$.

Une telle analyse contextualiste des concepts de couleur souligne le fait que la grammaire de ces concepts est nécessairement, pour reprendre le terme de Travis, «sensible à l'occasion $»^{2}$. Or, selon Travis, ce phénomène de sensibilité à l'occasion est «partout»" ${ }^{3}$. Plus exactement, la sensibilité à l'occasion concerne tous les concepts : «La sensibilité à l'occasion appartient au conceptuel $»^{4}$, nous dit-il. C'est l'usage des concepts qui est sensible à l'occasion. Deux points fondamentaux pour notre réflexion sont donc mis en avant. 1/ L'usage des concepts, de tous les concepts et non pas seulement des supposés concepts formels, est déterminé par des règles grammaticales ; $2 /$ ces règles ne sont pas elles-mêmes formelles et a priori mais sensibles à l'usage et à l'occasion.

Selon Travis, c'est précisément l'enseignement des 120 premiers paragraphes des Recherches de Wittgenstein. L'enjeu du concept de «jeu de langage » est par exemple de montrer que « la nomination ou la référence, même au sens large, sous-détermine les conditions de correction du tout, notamment, quand c'est pertinent, les conditions de sa vérité $»^{5}$. C'est également l'enjeu de toute la discussion que mène Wittgenstein sur la généralité des concepts depuis le Cahier bleu 6 , et qui aboutit à la discussion des « airs de famille » dans les paragraphes 65 à 92 des Recherches, que de souligner le

${ }^{1}$ Ch. Travis, «Jusqu'où va la raison», dans Id. Le silence des sens, Paris, Cerf, 2014, p. 201.

${ }^{2}$ Cf. Ch. Travis, Occasion-Sensitivity, Selected Essays, Oxford, Oxford University Press, 2008.

${ }^{3}$ Ibid., p. 1 .

${ }^{4}$ Ch. Travis, « Jusqu'où va la raison », art. cit., p. 206.

${ }^{5} \mathrm{Ch}$. Travis, « On Concepts of Objects », dans Occasion-sensitivity, op. cit., p. 254.

${ }^{6}$ Cf. L. Wittgenstein, Le Cahier bleu et le Cahier brun, Paris, Gallimard, 1996, p. 57. 
fait que l'application des concepts est sensible à l'usage, un usage toujours déterminé en contexte.

Or, ce que manifeste la grammaire, ce sont précisément les règles d'usage qui déterminent l'application des concepts. Reprenons l'un des exemples du dernier recueil de Travis traduit en français. Pour déterminer la manière dont je dois appliquer le concept de "viande », il faut encore déterminer ce qui compte comme de la viande pour l'usage que je veux en faire, dans un contexte donné : si notamment des « rognons » ou de la « viande en plastique » comptent pour de la viande ${ }^{1}$. En fonction de l'usage que je fais du concept, son application sera différemment réglée. Il sera par exemple incorrect, ou du moins non pertinent, de dire «ceci est de la viande " en désignant des rognons. Pour prendre d'autres exemples, pour déterminer si l'animal qu'a vu Pia est un "cochon», encore faut-il déterminer si «les marsupiaux des antipodes » ou les cochons en silicone comptent comme des cochons $^{2}$, pour décréter si l'eau du lac est «bleue», encore faut-il que l'on s'accorde pour savoir si je parle de l'eau du lac dans laquelle le beau ciel d'été se reflète ou de l'eau du lac que j'ai versée dans un verre transparent pour l'analyser ${ }^{3}$. Si vous avez très faim et que vous déclarez que n'importe quel « repas » ferait l'affaire, encore faut-il voir si la purée d'une fillette de deux ans compterait bien comme un repas. L'idée qui est sous-jacente à l'analyse, et que Travis retient de Wittgenstein, consiste donc à dire que tous les concepts fonctionnent ainsi : que tous les concepts, même les concepts prétendument logiques comme les concepts de couleur, sont sensibles à l'usage. C'est également vrai des concepts de «nombre». Rappelons par exemple que dans les rues de certaines villes, les nombres pairs désignent les maisons d'un côté de la rue et les nombres impairs les maisons de l'autre côté, alors que dans d'autres villes, la numérotation suit l'enfilade des maisons: les concepts de nombre y sont organisés différemment et leur usage diffère en conséquence en fonction de « l'occasion ».

Le rôle de la méthode grammaticale wittgensteinienne se précise en conséquence. L'enjeu de cette méthode est précisément d'être attentif à la sensibilité à l'usage et à l'occasion des règles qui gouvernent l'application des concepts. Comme le rappelle Wittgenstein dès le Tractatus (propositions 3.323 et 3.324), la méthode grammaticale a alors essentiellement une fonction thérapeutique, celle de lever les « confusions » ou «mécompréhensions

${ }^{1}$ Ch. Travis, Le silence des sens, op. cit., p. 200.

${ }^{2}$ Ibid., p. 81.

${ }^{3} \mathrm{Cf}$. Ch. Travis, Les liaisons ordinaires: Wittgenstein sur la pensée et le monde, Paris, Vrin, 2003, p. 75. 
relatives à l'usage des mots $»^{1}$. Sur ce point, il y a une profonde continuité dans les thèses de Wittgenstein. De telles confusions, nous précisaient déjà la proposition 3.323 du Tractatus et encore le $\S 16$ de la Grammaire philosophique, consistent à confondre deux mots qui « dénotent de manière différente $»^{2}$, par exemple, pour reprendre le $\S 16$ de la Grammaire, de confondre l'usage du concept «est» dans «la rose est rouge » et dans « $2 * 2=4$ ». Si on peut remplacer « est» par « est égal» dans « $2 * 2=4$ », c'est un non-sens de le faire pour « la rose est rouge ». Il appartient donc aux règles grammaticales de lever ces confusions, «en écartant les mécompréhensions relatives à l'usage des mots » :

$\S 90$. Nos considérations sont donc grammaticales. Et elles élucident notre problème en écartant les mécompréhensions relatives à l'usage des mots et provoqués notamment par certaines analogies entre les formes d'expression qui ont cours dans différents domaines de notre langage ${ }^{3}$.

Ces règles ont bien pour fonction de nous rendre attentifs à la sensibilité à l'usage et à l'occasion des concepts, un point auquel on se doit d'être attentif au risque de se méprendre sur le fonctionnement du langage.

Une ultime précision s'impose. Insister sur la sensibilité à l'occasion du conceptuel, ce n'est évidemment pas prêcher en faveur d'une thèse relativiste. Comme le dit très bien Travis dans l'introduction de son Occasion-sensitivy, " la relativité est une chose, la sensibilité à l'occasion en est une autre $»^{4}$. Les règles grammaticales, quoique non prescriptives, sont donc bien des règles comme ne cesse également de le rappeler Wittgenstein, des règles immanentes au pratiques : des règles d'usage censées trancher le sens du non-sens ou, si l'on préfère, le sens pertinent du non-sens impertinent. La thèse contextualiste héritée de l'analyse wittgensteinienne soutient de fait que les différents usages des concepts sont déterminés par des critères de correction, des critères fixés en contexte. Citons les Liaisons ordinaires de Travis: en circonstances, «il y a des usages que l'on peut raisonnablement attendre des mots [...] et d'autres dont ce n'est pas le cas $»^{5}$. Pour le dire autrement, une fois le contexte fixé, il y a nul doute possible quant à ce qui compte comme de la viande, un cochon, du bleu, de la purée ou le nombre 8 pour reprendre les exemples précédents. Il existe donc bien

${ }^{1}$ L. Wittgenstein, Recherches philosophiques, op. cit., § 90, p. 78.

${ }^{2}$ L. Wittgenstein, Grammaire philosophique, op. cit., p. 78.

${ }^{3}$ L. Wittgenstein, Recherches philosophiques, op. cit., p. 78.

${ }^{4}$ Ch. Travis, Occasion-sensitivity, op. cit., p. 13.

${ }^{5}$ Ch. Travis, Les liaisons ordinaires, op.cit., p. 150. 
un critère de pertinence pour distinguer le sens du non-sens : un critère d'usage dont les standards de correction sont fixés en contexte. Les règles grammaticales ne sont donc pas relatives à l'interprétation subjective qu'en fait tout un chacun. Elles sont déterminées, en contexte.

\section{Remarques conclusives : sémantique et syntaxe}

Nous avons commencé par insister, tout en la nuançant, sur une ressemblance possible entre le traitement husserlien des règles logiques de la grammaire pure et le traitement du Tractatus de la syntaxe logique. L'une et l'autre méthode, indépendamment de leurs divergences réelles, présentent le trait commun de clairement dissocier les plans de la morphologie du sens, de la logique de la vérité et de la référence objective. De ce point de vue, il apparaît cependant que le dit second Wittgenstein opère un pas supplémentaire.

De l'analyse dudit second Wittgenstein, nous retenons en effet que les règles grammaticales ne sont pas nécessairement formelles et que l'application des concepts n'est pas guidée par la «structure interne» des concepts. L'application de tous les concepts, nous montre Wittgenstein, est sensible à l'usage et au contexte. Aussi avons-nous mis l'accent sur un résultat radical : il résulte de l'analyse wittgensteinienne que la sémantique elle-même est réglée par des lois syntaxiques ou grammaticales. Comme l'affirme déjà Travis dans The Uses of Sense, «le facteur dont la sémantique d'un élément dépend », c'est « l'usage » ${ }^{1}$. Ces lois conditionnent jusqu'à l'application des concepts et, en conséquence, l'évaluation des jugements qui les mobilisent.

Il faut cependant s'entendre sur le sens à accorder à une telle conclusion. Il ne découle pas de la radicalité de cette thèse qu'il ne faut accorder aucun crédit aux règles syntaxiques classiques qui semblent gouverner l'ordonnancement de toutes les langues européennes. Comme le dit Wittgenstein lui-même, par exemple au $\S 498$ des Recherches, «lait-moi du sucre » n'est pas un énoncé correctement formé. Il semble même que Wittgenstein accorde une certain crédit à ce que Husserl appelait les "catégories de signification » (nom, verbe, sujet). Citons à ce titre la deuxième partie des Recherches de Wittgenstein : "Celui qui ne serait ni en état de dire que le mot "soit" peut être un verbe ou une conjonction, ni de construire des phrases dans lesquelles il a tantôt l'une, tantôt l'autre de ces fonctions, ne pourrait

${ }^{1}$ Ch. Travis, The Uses of Sense. Wittgenstein's Philosophy of Language, Oxford et New-York, Oxford University Press, 2001², p. 18. 
venir à bout de simples exercices scolaires $»^{1}$. Cependant, Wittgenstein ajoute une précision fondamentale à l'analyse en suggérant que ces dites "catégories de signification» sont elles-mêmes des catégories d'usage : "Qu'un signe "R" ou "N" soit "un mot ou une phrase" dépend de la situation dans laquelle il est prononcé ou écrit », pour reprendre le paragraphe 49 des Recherches $^{2}$. Le mot «Dalle!» par exemple peut être compris aussi bien comme une phrase que comme un mot, selon l'analyse des paragraphes 1920 des mêmes Recherches ${ }^{3}$. Il ne serait donc pas impensable d'être appelé à réviser les dites « catégories de signification » quand le contexte l'exige.

De plus, dire que la "sémantique est sensible à l'usage » ne signifie pas que l'on peut faire n'importe quel usage des concepts et leur faire dire ce que l'on veut. Pour reprendre encore des exemples de Wittgenstein, il est par exemple difficile de dire " "Ici, il fait froid", en voulant dire par là : "Ici il fait chaud" ${ }^{4}$. Il est clair que certains usages sont plus habituels que d'autres. Mais là encore, il s'agit exclusivement de règles grammaticales d'usage. C'est parce qu'il n'existe pas de contexte pertinent (du moins à notre connaissance) pour dire «Ici, il fait froid» en voulant dire par là : "Ici il fait chaud" que cela n'a pas de sens de le faire.

Ces précisions apportées, nous retenons de l'analyse wittgensteinienne que le partage classique entre syntaxe et sémantique doit vraisemblablement être repensé. Il n'est tout simplement plus possible d'opposer l'analyse formelle de la syntaxe du sens au calcul de vérité de la valeur des propositions. L'apport de la méthode grammaticale, nous semble-t-il, est de montrer que l'on ne peut déterminer la correction de l'application d'un concept qu'en regard des règles grammaticales d'usage, fixées en contexte. Car ce n'est qu'en contexte que l'on peut déterminer si l'on a correctement usé d'un concept en disant que c'est un cochon qui est dans la porcherie. Règles sémantiques et règles syntaxiques semblent en conséquence inextricablement liées, non pas parce que toute règle est sémantique - et que toute règle est une règle de correction mettant en jeu la vérité - mais bien plutôt parce que toute règle, qu'elle soit une règle de correction ou de vérité, est d'abord une règle d'usage grammatical.

${ }^{1}$ L. Wittgenstein, Recherches philosophiques II, t. ii, p. 249. Cf. ibid., p. 251.

${ }^{2}$ Ibid., $\S 49$, p. 55.

${ }^{3}$ Ibid., § 19-20, p. 35-36.

${ }^{4}$ Ibid., § 510, p. 201. 